\title{
Kidney Diseases Caused by Complement Dysregulation: Acquired, Inherited, and Still More to Come
}

\author{
Saskia F. Heeringa ${ }^{1}$ and Clemens D. Cohen ${ }^{2}$ \\ ${ }^{1}$ Division of Internal Medicine, University Hospital Zurich, Raemistrasse 100, 8006 Zurich, Switzerland \\ ${ }^{2}$ Division of Nephrology, University Hospital Zurich, Raemistrasse 100, 8006 Zurich, Switzerland
}

Correspondence should be addressed to Saskia F. Heeringa, saskia.heeringa@usz.ch

Received 6 August 2012; Accepted 11 October 2012

Academic Editor: Michael A. Flierl

Copyright (C) 2012 S. F. Heeringa and C. D. Cohen. This is an open access article distributed under the Creative Commons Attribution License, which permits unrestricted use, distribution, and reproduction in any medium, provided the original work is properly cited.

\begin{abstract}
Inherited and acquired dysregulation of the complement alternative pathway plays an important role in multiple renal diseases. In recent years, the identification of disease-causing mutations and genetic variants in complement regulatory proteins has contributed significantly to our knowledge of the pathogenesis of complement associated glomerulopathies. In these diseases defective complement control leading to the deposition of activated complement products plays a key role. Consequently, complement-related glomerulopathies characterized by glomerular complement component 3 (C3) deposition in the absence of local immunoglobulin deposits are now collectively described by the term "C3 glomerulopathies." Therapeutic strategies for reestablishing complement regulation by either complement blockade with the anti-C5 monoclonal antibody eculizumab or plasma substitution have been successful in several cases of C3 glomerulopathies. However, further elucidation of the underlying defects in the alternative complement pathway is awaited to develop pathogenesis-specific therapies.
\end{abstract}

\section{Introduction}

The central function of the kidney for whole body homeostasis is based on adequate blood flow and pressure, sufficient glomerular capillary surface for selective filtration, and subsequent secretion and reabsorption of solutes in the tubular system. The essential role of the glomerulus as a filtration unit can be estimated by the fact that most diseases leading to chronic kidney disease and end-stage renal disease with the need for dialysis or transplantation are caused by glomerulopathies. The glomerulus as a specialized capillary convolute is prone to any vascular damage and is affected as part of a generalized microangiopathy in common diseases such as diabetes mellitus or arterial hypertension. However, the glomerulus can also be affected by specific circulating factors, including antibodies against glomerular antigens, circulating immune complexes, or activated factors of a dysregulated complement system.

The complement system as an essential component of the innate immune system plays an indispensable role in the elimination of invading microorganisms as a first line of defense $[1,2]$. Furthermore, the complement system bridges innate and adaptive immunity. The cross-talk between toll-like receptors-as another key component of the innate immune system-and the complement system has been a key aspect of research as of their synergistic interaction to increase activation of inflammatory responses [3]. Complement activation runs through three major pathways (classic, alternative, and mannose-binding lectin) that all generate the enzyme complex $\mathrm{C} 3$-convertase which cleaves C3 into C3a and C3b. Herein, four main activation steps are distinguished: initiation of activation, activation and amplification of C3-convertase, activation of C5-convertase, and activation of the terminal pathway activity which is characterized by the assembly of the membrane attack complex (MAC) [4]. Importantly, the alternative pathway is constantly activated at low levels. Cascade progression and activation, however, is strictly controlled by complement regulating proteins such as complement factor $\mathrm{H}(\mathrm{CFH})$ and complement factor I (CFI): the two most important inhibitory proteins of the alternative pathway. 


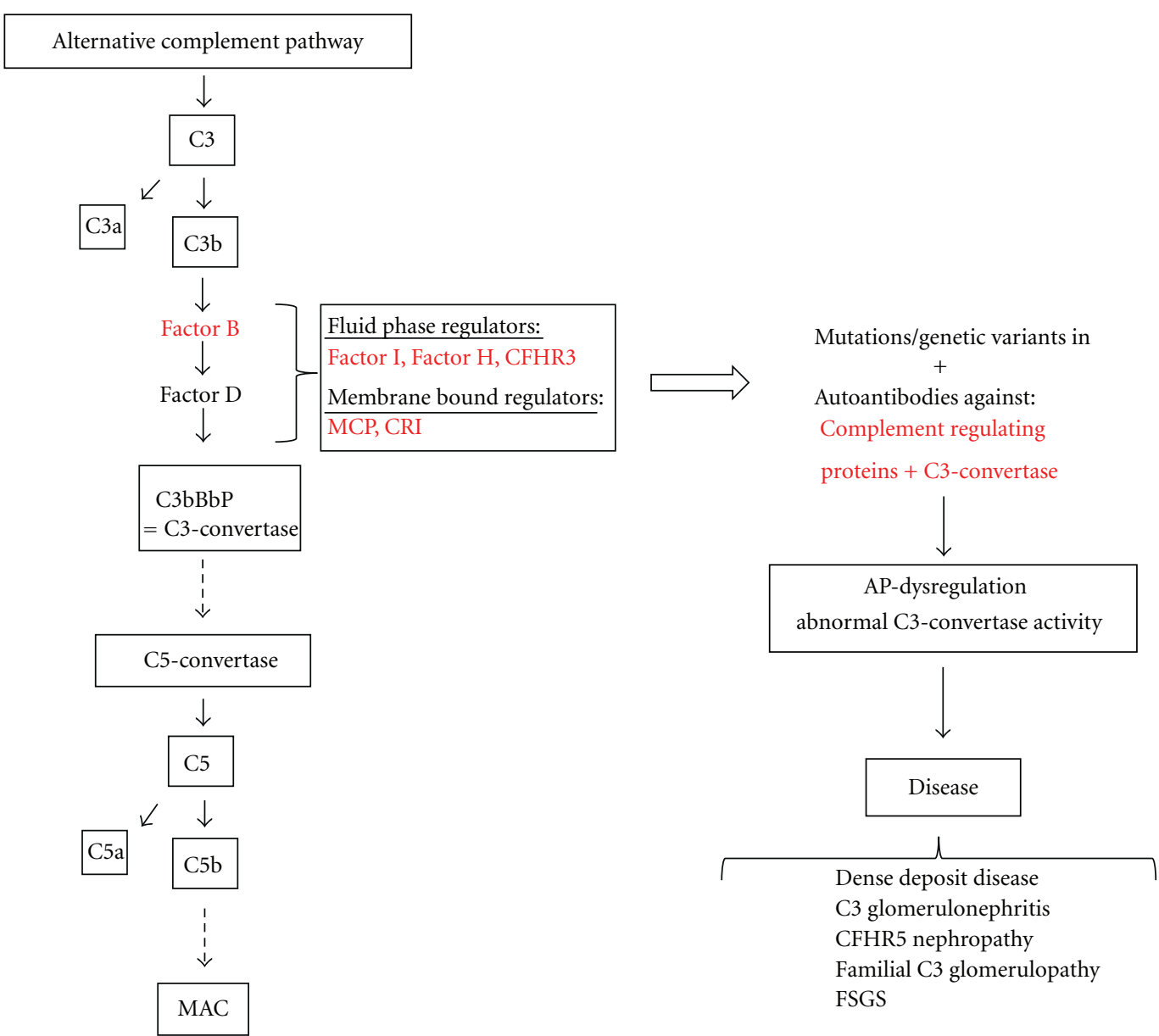

Figure 1: Dysregulation of the alternative complement cascade due to acquired or genetic factors leads to defective complement control causing a range of complement-associated glomerulopathies. C3 is cleaved to generate C3a and C3b. After binding of C3b to factor B, the complex is cleaved by factor D to form C3-convertase. The initial convertase constantly cleaves C3 at a low rate (referred to as "tick-over" of the alternative pathway). Binding of another C3b-fragment to C3-convertase creates a C5-convertase after which the pathway proceeds in the same manner as the classical pathway recruiting additional complement factors to ultimately form the membrane attack complex (MAC). The alternative pathway is strictly regulated by complement regulating proteins (listed in red). Mutations, genetic variations, or antibodies against complement regulating proteins or C3-convertase lead to abnormal C3-convertase activity. The subsequent deposition of activated complement products causes a range of complement-associated glomerulopathies. Abbreviations: C3; complement component 3, CFHR3; complement factor $\mathrm{H}$ related protein, AP; alternative pathway.

Complement dysregulation has been early recognized to be a central event in many nephropathies, and peripheral markers for complement activation (especially serum levels of $\mathrm{C} 3$ and $\mathrm{C} 4)$ are tested routinely for different acquired renal diseases, for example, postinfectious glomerulopathy and proliferative lupus nephritis, glomerular capillaritis due to cryoglobulinemia or cholesterol embolism. Moreover, an increasing number of inherited renal diseases and renal diseases due to acquired factors with genetic predisposition for complement dysregulation are discovered such as atypical hemolytic uremic syndrome (aHUS) and membranoproliferative glomerulonephritis (MPGN) forms including dense deposit (DDD), C3 glomerulonephritis (C3GN) and CFHR5 nephropathy (Figure 1) $[5,6]$. Mutations in $C F H$ leading to CFH dysfunction and subsequently aHUS are the best known disease-causing mutations, but mutations in several other genes coding for complement factors and regulatory proteins have been identified in complementrelated glomerulopathies (e.g., C3, CFI, CFHR1-5, MCP (membrane cofactor protein)). Genome-wide linkage analysis recently added novel polymorphisms and diseasecausing mutations in complement genes to the list of hereditary complement-related nephropathies $[7,8]$. In the following minireview we give an overview of complementrelated glomerulopathies. Atypical HUS, a syndrome with prominent nonrenal, that is hematological and neurological manifestations, will not be discussed.

\section{C3 Glomerulopathy}

Patients with MPGN due to complement dysregulation manifest with proteinuria, (micro)hematuria and a variable degree of renal insufficiency. Kidney biopsy results show an altered glomerular basement membrane with double 
TABLE 1: C3 glomerulopathies.

\begin{tabular}{|c|c|c|c|}
\hline Diseases & EM-findings & Alternative pathway abnormalities & Disease specific treatment options \\
\hline Dense deposit disease & $\begin{array}{l}\text { (i) Osmophilic wavy dense } \\
\text { deposits within GBM, } \\
\text { mesangial matrix, tubular BM }\end{array}$ & $\begin{array}{l}\text { (i) Autoantibodies (C3Nef, } \\
\text { FHAA, FBAA, C3-convertase } \\
\text { AA) } \\
\text { (ii) Mutations/genetic variations } \\
(C F H, C F I, C F B, M C P)\end{array}$ & $\begin{array}{l}\text { (i) Infusion of fresh frozen plasma } \\
\text { (ii) Plasmapheresis } \\
\text { (iii) Eculizumab } \\
\text { (iv) Immunosuppressive } \\
\text { treatment (in case of } \\
\text { autoantibodies) }\end{array}$ \\
\hline C3 glomerulonephritis & $\begin{array}{l}\text { (i) Mesangial, subendothelial, } \\
\text { subepithelial and } \\
\text { intramembranous deposits }\end{array}$ & $\begin{array}{l}\text { (i) Mutations/genetic variations } \\
(C F H, C F I, M C P) \\
\text { (ii) Autoantibodies (C3Nef, } \\
\text { FHAA) }\end{array}$ & $\begin{array}{l}\text { (i) Eculizumab } \\
\text { (ii) Immunosuppressive treatment } \\
\text { (in case of autoantibodies) }\end{array}$ \\
\hline CFHR5 nephropathy & $\begin{array}{l}\text { (i) Mesangial, subendothelial, } \\
\text { subepithelial deposits }\end{array}$ & (i) CFHR5-mutation & $\begin{array}{l}\text { (i) No treatment of proven efficacy } \\
\text { (ii) Plasma exchange associated } \\
\text { with good outcome }\end{array}$ \\
\hline $\begin{array}{l}\text { Familial C3 } \\
\text { glomerulopathy }\end{array}$ & $\begin{array}{l}\text { (i) MPGN type III } \\
\text { (ii) Subendothelial, subepithelial } \\
\text { deposits }\end{array}$ & $\begin{array}{l}\text { (i) Familial hybrid CFHR3-1 gene } \\
\text { autosomal dominant } \\
\text { inheritance }\end{array}$ & (i) No treatment of proven efficacy \\
\hline
\end{tabular}

Abbreviations: C3: complement component 3, CFHR5: complement factor H related protein 5, CFH: complement factor H, CFI: complement factor I, MCP: membrane cofactor protein, FHAA: factor H autoantibody, FBAA: factor B autoantibody, (G) BM; (glomerular) basement membrane, MCP: membrane cofactor protein.

contours mostly due to subendothelial deposits, besides a variety of additional alterations such as hypercellularity and additional deposits [6]. As MPGN can be immunecomplex-mediated, specific immunofluorescence has to be employed when evaluating the renal biopsy to differentiate between immunglobulin-mediated MPGN and complement-mediated MPGN.

Based on the localization of deposits in electron microscopy, MPGN has been classified into three different types: type I (subendothelial deposits), type II (intramembranous deposits), and type III (subendothelial and subepithelial deposits) [9]. Type I and III typically are immunglobulin-mediated diseases caused by the deposition of immune complexes as a result of for example circulating immune complexes, monoclonal gammopathies or chronic infections. MPGN II which is also called dense deposit disease, is characterized by complement component 3 (C3) containing dense deposits in the glomerular basement membrane that are a result of a dysregulation of the complement alternative pathway. As the inflammation is caused directly by the deposition of complement products, immunoglobulins are not involved and therefore not observed in immunofluorescence studies [6].

The subgrouping of MPGN has led to some confusion as all types of MPGN stain positive for C3 but immunglobulin staining can be negative even in some cases of MPGN I and III. Sethi et al. therefore proposed a classification driven by the findings on immunofluorescence, classifying MPGN as either immunoglobulin positive or negative [10]. Hence, immunoglobulin-negative but C3-positive MPGN is newly referred to as C3 glomerulopathy. Examples of C3 glomerulopathies are C3GN and DDD that can be distinguished by electron microscopical findings (see Table 1).

C3GN appears to be a key example of a dysregulated alternative and terminal complement pathway in which the deposition of complement is triggered despite the absence of antibody deposition $[5,10,11]$. Besides the identification of several disease-causing mutations in alternative pathway inhibitors, some autoantibodies leading to the activation or blockage of alternative pathway proteins have also been identified as a cause of C3GN. In a recent study by Servais et al., patients with C3GN (and additional patients with other forms of MPGN) were screened for mutations and rare variants in $C F H, C F I$, and $M C P$ [12]. Although genetic abnormalities found in patients with C3GN were similar to the ones reported in individuals affected by aHUS, the genetic background predisposes specifically for the respective clinical and histological phenotype [12]. A rare, recently described variant of C3GN is CFHR5 nephropathy, a monogenic disease caused by mutations in the gene encoding complement factor-related protein 5 (CFHR5) [7]. CFHR5 is structurally related to $\mathrm{CFH}$ and possibly acts as a cofactor inhibiting C3-convertase [13]. In a cohort of patients with familial CFHR5 nephropathy sharing the same founder mutation, it was shown that the phenotype-spectrum among family members is broad [14]. As of this phenotypic heterogeneity, it is assumed that other factors like predisposing modifier genes and environmental factors as complementactivating infections also play a role in the development and phenotype of disease. CFHR5 is a member of the CFH related protein family, consisting of 5 proteins; CFHR1-5. Little is known about the function of these proteins, but there is increasing evidence that these protein families may either be involved in disease development or protection from complement dysregulation, respectively. CFHR1 inhibits C5-convertase activity and the formation of the terminal complex. CFHR3 also has complement regulatory activity as it inhibits C3-convertase [15]. Interestingly, a complete absence of both genes ( $\triangle$ CFHR3-1) is not uncommon in the normal population. The deletion of CFHR3-1 has even been associated with protection from both complement- and age-related macular degeneration as well as IgA nephropathy, 
the most common mesangioproliferative glomerulonephritis with prominent mesangial IgA and secondary local complement activation [16]. In a recent study, a hybrid CFHR3-1 gene was shown to cause familial C3 glomerulopathy [17]. The authors suggested a possible dominant mechanism of this genetic alteration leading to an increased expression of both proteins, interfering with complement processing and leading to accumulation of C3 [17].

Dense deposit disease (DDD) is closely related to C3GN and recent data suggest that both may represent extremes in a continuous spectrum of complement-related MPGNs $[6,12]$. Both diseases show similar features in light and immunofluorescence microscopy and they are distinguished by electron microscopy. Here, C3GN is characterized by mesangial, subendothelial, and intramembranous deposits, whereas DDD is characterized by osmophilic dense deposits along the glomerular and tubular basement membranes $[18,19]$. By an advanced mass spectrometry approach on glomerular isolates, Sethi et al. detected activated components of the alternative pathway and of the terminal complement pathway in patients with DDD [20]. Because of a very high recurrence rate after kidney transplantation, a systemic cause of DDD has been early suggested. Hence, the identification of the first C3 nephritic factor (C3Nef) as an autoantibody that stabilizes $\mathrm{C} 3$-convertase, was a major achievement [21]. The presence of C3Nefs is the most common association with alternative pathway dysregulation in DDD [22]. Binding of C3Nef to the alternative C3convertase increases its half-life leading to uncontrolled alternative pathway activation and a massive consumption of C3. However, C3Nef activity is not always associated with low C3 levels in plasma and C3Nef is not specific for DDD as it can be found in other diseases as well as in healthy individuals [23]. Less common causes for DDD are inhibitory CFH autoantibodies, CFH deficiency, or functional $\mathrm{CFH}$-defects, the latter both due to genetic mutations leading to reduced $\mathrm{CFH}$ activity. Mutations that lead to a complete $\mathrm{CFH}$ deficiency are rare though, and most functional $\mathrm{CFH}$ defects go along with normal $\mathrm{CFH}$ levels in plasma $[24,25]$. Interestingly, not all patients with a functional CFH defect develop DDD, as CFH deficiency can also lead to aHUS and not all individuals with similar genetic variants develop the same phenotype [8].

In order to analyze the causes of alternative pathway dysregulation, several groups studied DDD cohorts employing functional and genetic tests $[12,22]$. In these studies, a probable cause for complement dysregulation could be identified in about $80 \%$ of DDD patients $[12,22]$. Detection of $\mathrm{C} 3 \mathrm{Nef}$ was the most frequent single finding, but in some patients also autoantibodies against $\mathrm{CFH}$ or $\mathrm{CFB}$ were detected [22]. Gene variants in CFH were detected in 10$17 \%$ of DDD patients $[12,22]$. Notably, a functional CFH defect frequently coexisted with the presence of C3Nef. Similarly, in C3GN the detection of C3Nef was the most common complement abnormality found in $45 \%$ patients with C3GN that were screened by Servais et al. [12]. Besides C3Nef, additional autoantibodies against $\mathrm{CFH}, \mathrm{CFB}$ and to the individual components of $\mathrm{C} 3$-convertase $(\mathrm{C} 3 \mathrm{~b}$ and factor B) have been described in C3 glomerulopathies
[12, 18, 26-28]. Anti-factor B autoantibody for example was found in a patient with DDD that was able to bind and thereby stabilize C3-convertase leading to an increased consumption of C3 [28]. This again indicates that DDD and C3GN have many pathogenetic and histological aspects in common and may represent extremes of a continuum.

\section{Treatment}

In order to decrease proteinuria and improve blood pressure control, nonspecific treatment with angiotensin converting enzyme (ACE) inhibitors or angiotensin type II receptor blockers is recommended in all patients. With improved understanding of the pathogenesis of C3 glomerulopathies more specific therapies could be applied. In patients with C3Nef or autoantibodies to CFH or CFB immunosuppressive therapies including rituximab or plasma exchange have been reported to slow disease progression or to help to avoid recurrence after transplantation $[19,26]$.

With the increasing knowledge about the underlying mechanisms and the specific complement defects in C3 glomerulopathies, specific complement-targeting therapies have been successfully applied in several cases of DDD and C3GN. As can be expected, large-scale clinical studies are missing in these rare diseases. Especially eculizumab, a humanized anti-C5 monoclonal antibody, represents a promising agent as it blocks C5b-9 formation, the terminal event in the complement cascade. The antibody has been approved by both the U.S. Food and Drug Administration as well as the European Commission for the treatment of paroxysmal nocturnal hemoglobinuria and, more recently, atypical HUS. Eculizumab was even suggested to be an effective agent in children with enterohemorrhagic Escherichia coli (EHEC) infection-caused HUS [29]. However, this was not evident in a case-control study reporting a mainly middle-aged population of a recent outbreak of EHEC induced HUS in northern Germany [30]. But for DDD and MPGN with CFHR1 deficiency, recent anecdotic reports suggest a treatment effect of eculizumab with stabilization of kidney function, decrease in proteinuria $[31,32]$, and even improvement in histopathological findings [33]. In a recent study reporting of three subjects with DDD and three subjects with C3GN who were treated with eculizumab for one year, response to treatment was seen in some but not all patients. Elevated serum membrane attack complex normalized on therapy, serum creatinine improved and proteinuria was reduced [34]. A very recent pathology report on C3GN patients treated with eculizumab showed de-novo monoclonal staining for IgG-kappa in the same distribution as C3 and C5b9 in all posttreatment protocol biopsies, indicating binding to C5 and glomerular deposition of eculizumab [35]. As the authors state, the long-term clinical significance of these therapy-induced immune deposits together with apparent drug-tissue interactions is not known. Beside eculizumab as target-specific but costly treatment option fresh frozen plasma (FFP) infusions were given to several cases with functional $\mathrm{CFH}$ deficits and resulted in the prevention 
of further disease progression $[36,37]$. In a recent case report of two unrelated patients with MPGN and MPGN II with combined autoantibodies for factor B and C3, one patient received immunosuppressive treatment leading to a significant decrease of both autoantibodies [26].

In order to guide such disease specific treatment, it may become important to evaluate the alternative complement pathway more comprehensively in patients that have a renal biopsy consistent with C3 glomerulopathy. Complement proteins ( $\mathrm{CFH}, \mathrm{CFI}, \mathrm{CFB})$, complement degradation products $(\mathrm{C} 3 \mathrm{c}, \mathrm{C} 3 \mathrm{~d})$, soluble serum membrane attack complex (sMAC), and disease associated autoantibodies (C3 nephritic factor, anti-factor $\mathrm{H}$, anti-factor $\mathrm{B}$, anti-C3b [26]) might expand the diagnostic arsenal of complement specific markers in the future.

\section{Conclusions}

In sum, the spectrum of renal disease phenotypes due to complement dysregulation is diverse. And it is still increasing: in a recent case report, Sethi et al. reported of single nucleotide polymorphisms in genes encoding $\mathrm{CFH}$ and $\mathrm{C} 3$ to be linked to the development of focal segmental glomerulosclerosis (FSGS), potentially extending the involvement of complement dysregulation to "podocytopathies" [38, 39]. Interestingly, this finding is in line with data from the European Renal cDNA Bank indicating alteration of intraglomerular transcript levels of complement-related gene products in FSGS (own unpublished observation).

As outlined above, several independent approaches such as Mendelian genetics, genome-wide association studies, transcriptomic and proteomic approaches as well as histopathology and functional studies underline the relevance of complement dysregulation in several kidney diseases, which currently undergo a redefinition. With the increasing insight into the pathophysiology, more specific complement targeting therapies may become available for the treatment of both ultrarare and more frequent complement-associated renal diseases.

\section{Acknowledgments}

C. D. Cohen was supported by the Else Kröner-Fresenius Foundation and the Swiss National Science Foundation in frame of the National Center of Competence in Research: Kidney-Control of Homeostasis (NCCR Kidney.CH).

\section{References}

[1] M. J. Walport, "Advances in immunology: complement (first of two parts)," The New England Journal of Medicine, vol. 344, no. 14, pp. 1058-1066, 2001.

[2] M. J. Walport, "Advances in immunology: complement (second of two parts)," The New England Journal of Medicine, vol. 344, no. 15, pp. 1140-1144, 2001.

[3] B. Holst, A.-C. Raby, J. E. Hall, and M. O. Labéta, "Complement takes its Toll: an inflammatory crosstalk between Toll-like receptors and the receptors for the complement anaphylatoxin C5a," Anaesthesia, vol. 67, no. 1, pp. 60-64, 2012.

[4] P. F. Zipfel and C. Skerka, "Complement regulators and inhibitory proteins," Nature Reviews Immunology, vol. 9, no. 10, pp. 729-740, 2009.

[5] F. Fakhouri, V. Frémeaux-Bacchi, L. H. Noël, H. T. Cook, and M. C. Pickering, "C3 glomerulopathy: a new classification," Nature Reviews Nephrology, vol. 6, no. 8, pp. 494-499, 2010.

[6] S. Sethi and F. C. Fervenza, "Membranoproliferative glomerulonephritis-a new look at an old entity," The New England Journal of Medicine, vol. 366, no. 12, pp. 1119-1131, 2012.

[7] D. P. Gale, E. G. de Jorge, H. T. Cook et al., "Identification of a mutation in complement factor $\mathrm{H}$-related protein 5 in patients of Cypriot origin with glomerulonephritis," The Lancet, vol. 376, no. 9743, pp. 794-801, 2010.

[8] J. Caprioli, P. Bettinaglio, P. F. Zipfel et al., "The molecular basis of familial hemolytic uremic syndrome: mutation analysis of factor $\mathrm{H}$ gene reveals a hot spot in short consensus repeat 20," Journal of the American Society of Nephrology, vol. 12, no. 2, pp. 297-307, 2001.

[9] E. C. Jackson, A. J. McAdams, C. F. Strife, J. Forristal, T. R. Welch, and C. D. West, "Differences between membranoproliferative glomerulonephritis types I and III in clinical presentation, glomerular morphology, and complement perturbation," American Journal of Kidney Diseases, vol. 9, no. 2, pp. 115-120, 1987.

[10] S. Sethi, C. M. Nester, and R. J. H. Smith, "Membranoproliferative glomerulonephritis and C3 glomerulopathy: resolving the confusion," Kidney International, vol. 81, no. 5, pp. 434441, 2012.

[11] S. Sethi, F. C. Fervenza, Y. Zhang et al., "Proliferative glomerulonephritis secondary to dysfunction of the alternative pathway of complement," Clinical Journal of the American Society of Nephrology, vol. 6, no. 5, pp. 1009-1017, 2011.

[12] A. Servais, L.-H. Noël, L. T. Roumenina et al., "Acquired and genetic complement abnormalities play a critical role in dense deposit disease and other C3 glomerulopathies," Kidney International, vol. 82, no. 4, pp. 454-464, 2012.

[13] J. L. McRae, T. G. Duthy, K. M. Griggs et al., "Human factor Hrelated protein 5 has cofactor activity, inhibits C3 convertase activity, binds heparin and C-reactive protein, and associates with lipoprotein," Journal of Immunology, vol. 174, no. 10, pp. 6250-6256, 2005.

[14] Y. Athanasiou, K. Voskarides, D. P. Gale et al., "Familial C3 glomerulopathy associated with CFHR5 mutations: clinical characteristics of 91 patients in 16 pedigrees," Clinical Journal of the American Society of Nephrology, vol. 6, no. 6, pp. 14361446, 2011.

[15] L. G. Fritsche, N. Lauer, A. Hartmann et al., "An imbalance of human complement regulatory proteins CFHR1, CFHR3 and factor $\mathrm{H}$ influences risk for age-related macular degeneration (AMD)," Human Molecular Genetics, vol. 19, no. 23, pp. 46944704, 2010.

[16] A. G. Gharavi, K. Kiryluk, M. Choi et al., "Genomewide association study identifies susceptibility loci for IgA nephropathy," Nature Genetics, vol. 43, no. 4, pp. 321-327, 2011.

[17] T. H. Malik, P. J. Lavin, E. G. De Jorge et al., "A hybrid CFHR3-1 gene causes familial C3 glomerulopathy," Journal of the American Society of Nephrology, vol. 23, no. 7, pp. 11551160, 2012.

[18] S. Sethi, F. C. Fervenza, Y. Zhang et al., "C3 glomerulonephritis: clinicopathological findings, complement abnormalities, 
glomerular proteomic profile, treatment, and follow-up," Kidney International, vol. 82, no. 4, pp. 465-473, 2012.

[19] R. J. H. Smith, C. L. Harris, and M. C. Pickering, "Dense deposit disease," Molecular Immunology, vol. 48, no. 14, pp. 1604-1610, 2011.

[20] S. Sethi, J. D. Gamez, J. A. Vrana et al., "Glomeruli of dense deposit disease contain components of the alternative and terminal complement pathway," Kidney International, vol. 75, no. 9, pp. 952-960, 2009.

[21] R. E. Spitzer, E. H. Vallota, J. Forristal et al., "Serum C'3 lytic system in patients with glomerulonephritis," Science, vol. 164, no. 3878, pp. 436-437, 1969.

[22] Y. Zhang, N. C. Meyer, K. Wang et al., "Causes of alternative pathway dysregulation in dense deposit disease," Clinical Journal of the American Society of Nephrology, vol. 7, no. 2, pp. 265-274, 2012.

[23] A. T. Gewurz, S. M. Imherr, S. Strauss, H. Gewurz, and C. Mold, "C3 nephritic factor and hypocomplementaemia in a clinically healthy individual," Clinical and Experimental Immunology, vol. 54, no. 1, pp. 253-258, 1983.

[24] T. H. J. Goodship, I. Y. Pappworth, T. Toth et al., "Factor H autoantibodies in membranoproliferative glomerulonephritis," Molecular Immunology, vol. 52, no. 3-4, pp. 200-206, 2012.

[25] C. Licht, U. Schlötzer-Schrehardt, M. Kirschfink, P. F. Zipfel, and B. Hoppe, "MPGN II-genetically determined by defective complement regulation?" Pediatric Nephrology, vol. 22, no. 1, pp. 2-9, 2007.

[26] Q. Chen, D. Müller, B. Rudolph et al., "Combined C3b and factor B autoantibodies and MPGN type II," The New England Journal of Medicine, vol. 365, no. 24, pp. 2340-2342, 2011.

[27] T. S. Jokiranta, A. Solomon, M. K. Pangburn, P. F. Zipfel, and S. Meri, "Nephritogenic $\lambda$ light chain dimer: a unique human miniautoantibody against complement factor H," Journal of Immunology, vol. 163, no. 8, pp. 4590-4596, 1999.

[28] S. Strobel, M. Zimmering, K. Papp, J. Prechl, and M. Józsi, "Anti-factor B autoantibody in dense deposit disease," Molecular Immunology, vol. 47, no. 7-8, pp. 1476-1483, 2010.

[29] L. Anne-Laure, M. Malina, V. Fremeaux-Bacchi et al., "Eculizumab in severe shiga-toxin-associated HUS," The New England Journal of Medicine, vol. 364, no. 26, pp. 25612563, 2011.

[30] J. Menne, M. Nitschke, R. Stingele et al., "Validation of treatment strategies for enterohaemorrhagic Escherichia coli O104:H4 induced haemolytic uraemic syndrome: case-control study," British Medical Journal, vol. 345, no. 7869, Article ID e4565, 2012.

[31] E. Daina, M. Noris, and G. Remuzzi, "Eculizumab in a patient with dense-deposit disease," The New England Journal of Medicine, vol. 366, no. 12, pp. 1161-1163, 2012.

[32] S. Radhakrishnan, A. Lunn, M. Kirschfink et al., "Eculizumab and refractory membranoproliferative glomerulonephritis," The New England Journal of Medicine, vol. 366, no. 12, pp. 1165-1166, 2012.

[33] M. Vivarelli, A. Pasini, and F. Emma, "Eculizumab for the treatment of dense-deposit disease," The New England Journal of Medicine, vol. 366, no. 12, pp. 1163-1165, 2012.

[34] A. S. Bomback, R. J. Smith, G. R. Barile et al., "Eculizumab for dense deposit disease and C3 glomerulonephritis," Clinical Journal of the American Society of Nephrology, vol. 7, no. 5, pp. 748-756, 2012.

[35] L. C. Herlitz, A. S. Bomback, G. S. Markowitz et al., "Pathology after eculizumab in dense deposit disease and C3 GN," Journal of the American Society of Nephrology, vol. 23, no. 7, pp. 12291237, 2012.

[36] S. Habbig, M. J. Mihatsch, S. Heinen et al., "C3 deposition glomerulopathy due to a functional factor H defect," Kidney International, vol. 75, no. 11, pp. 1230-1234, 2009.

[37] C. Licht, A. Weyersberg, S. Heinen et al., "Successful plasma therapy for atypical hemolytic uremic syndrome caused by factor $\mathrm{H}$ deficiency owing to a novel mutation in the complement cofactor protein domain 15," American Journal of Kidney Diseases, vol. 45, no. 2, pp. 415-421, 2005.

[38] S. Sethi, F. C. Fervenza, Y. Zhang, and R. J. H. Smith, "Secondary focal and segmental glomerulosclerosis associated with single-nucleotide polymorphisms in the genes encoding complement factor $\mathrm{H}$ and C3," American Journal of Kidney Diseases, vol. 60, no. 2, pp. 316-321, 2012.

[39] R. C. Wiggins, "The spectrum of podocytopathies: a unifying view of glomerular diseases," Kidney International, vol. 71, no. 12, pp. 1205-1214, 2007. 


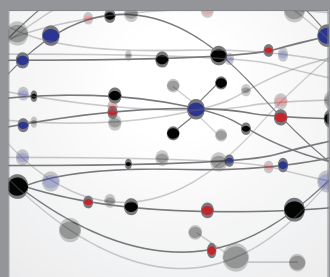

The Scientific World Journal
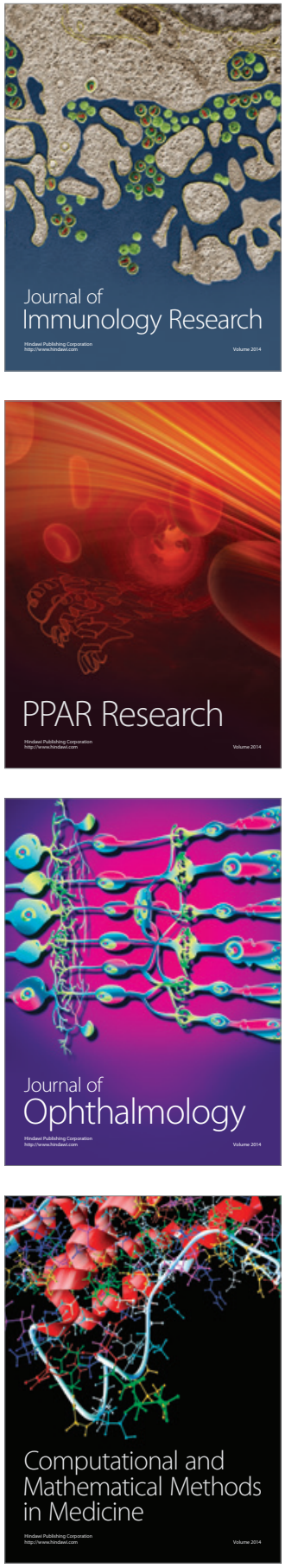

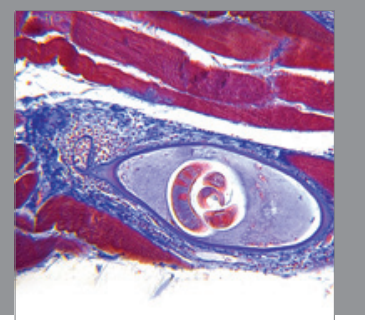

Gastroenterology

Research and Practice
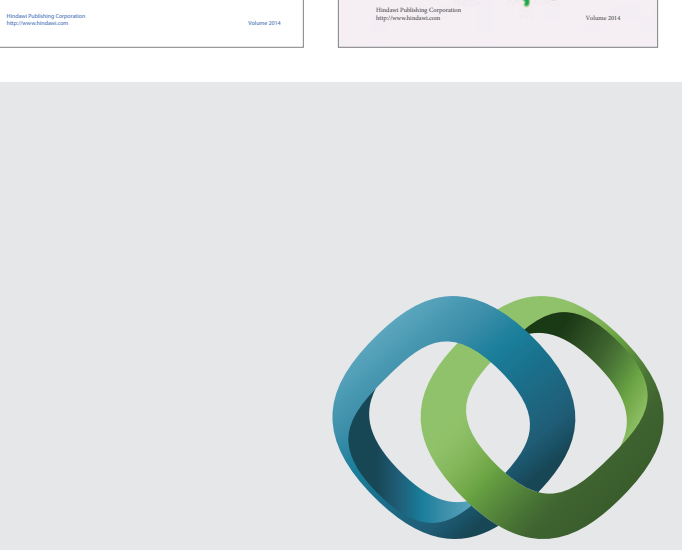

\section{Hindawi}

Submit your manuscripts at

http://www.hindawi.com
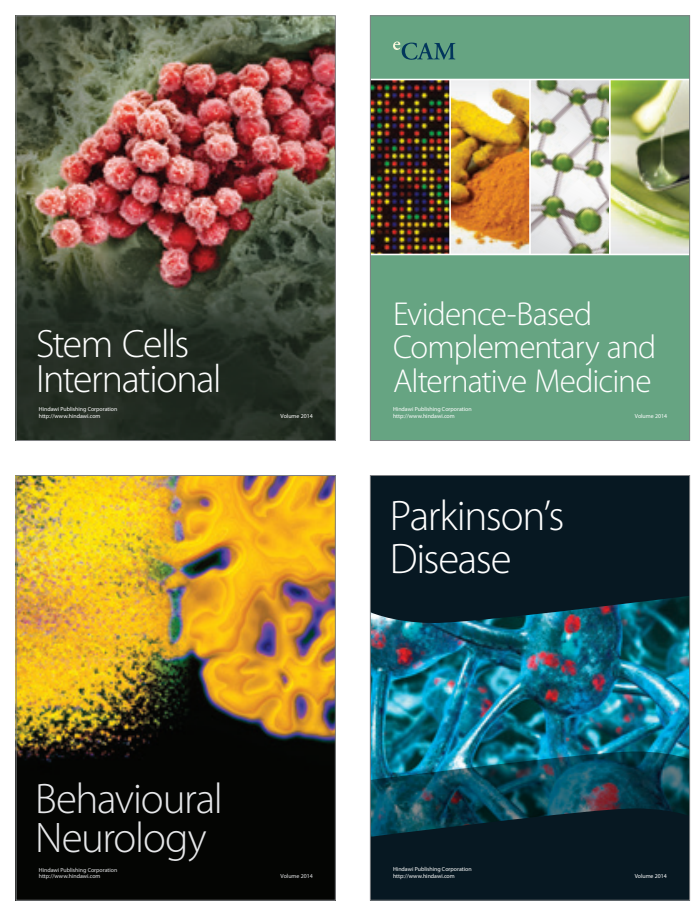

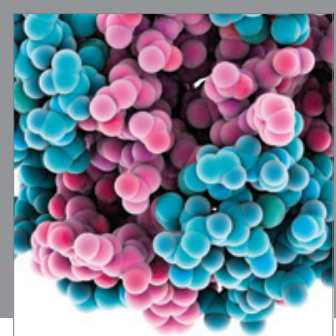

Journal of
Diabetes Research

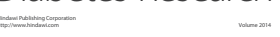

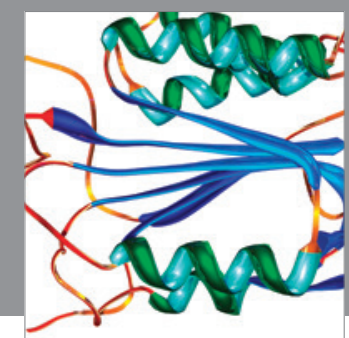

Disease Markers
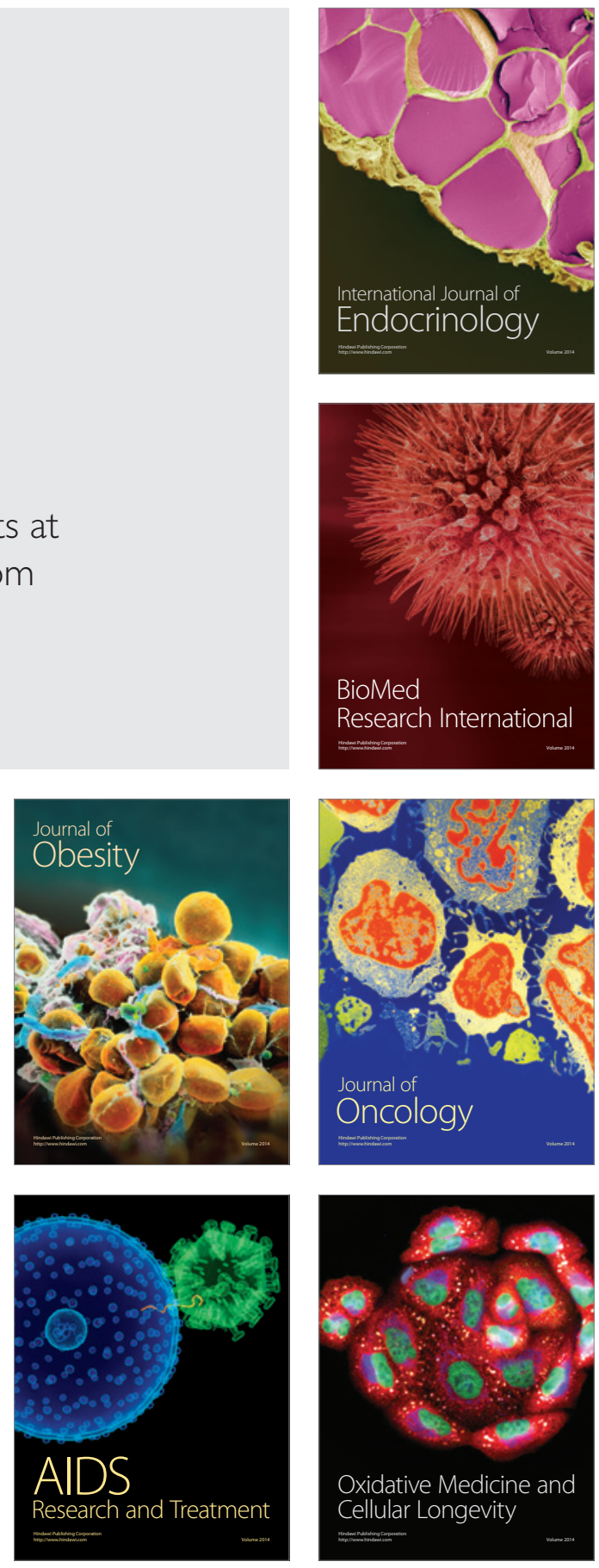